\title{
The impact of the errors of collinear functions in describing unintegrated SIDIS data
}

\author{
Andrea Simonelli* \\ University of Turin, Italy and INFN, Sezione di Torino \\ E-mail: andrea.simonellieunito.it

\section{Mariaelena Boglione} \\ Affiliation: University of Turin, Italy and INFN, Sezione di Torino \\ E-mail: boglioneeto.infn.it

\section{Osvaldo Gonzalez} \\ Affiliation: University of Turin, Italy and INFN, Sezione di Torino \\ E-mail: joghdregmail.com
}

Describing $q_{T}$-dependent SIDIS distributions requires consideration of two different regimes which involve two different factorization schemes. In both cases, the necessary ingredients include parton distribution and fragmentation functions (PDFs and FFs), which are traditionally extracted in statistical analyses with collinear observables. We will present examples of how the errors in the extraction of collinear PDFs and FFs may affect the successful description of the $q_{T}$-dependent SIDIS distributions.

XXVII International Workshop on Deep-Inelastic Scattering and Related Subjects - DIS2019 8-12 April, 2019

Torino, Italy

* Speaker. 


\section{Introduction and theoretical background}

Hadronic processes involving the production of jets at high energies can be studied in the framework of factorization [1], which allows to distinguish long-distance (low-energy) effects from short-distance (high-energy) contributions. The first are usually parametrized by suitable sets of phenomenological universal quantities, as Parton Distributions (PDFs) and Fragmentation Functions (FFs), while the latter are computable in perturbative QCD. In order to obtain a reliable picture of how confinement works, the role of the non-perturbative parts is crucial in the attempt to describe data. The correct parametrization in terms of PDFs and FFs depends on whether the partons associated to the jets have a transverse momentum that can be neglected. When this is the case, the non-perturbative functions are called collinear, since they do not account for partonic transverse motion. Instead, when parton transverse momenta cannot be neglected, we rely on Transverse Momentum Dependent (TMD) parton distributions. Two distinct schemes of factorization have to be applied in these two cases.

We will focus on SIDIS (Semi-Inclusive Deep Inelastic Scattering). This process consists in an initial state, associated with the incoming hadron described by a PDF, and a final state, generated by one outgoing hadron and related to a FF. The choice of factorization scheme depends on the ratio between the magnitude of the transverse momentum $q_{T}$ of the virtual photon that mediates the interaction and the transferred momentum $Q$ in the c.m. frame. It can be shown [1] that the collinear scheme applies in the large- $q_{T}$ region $\left(q_{T} / Q \gg 1\right)$, while in the low- $q_{T}$ region $\left(q_{T} / Q \ll 1\right)$ the TMD factorization scheme has to be used. Anyway, independently of the chosen factorization scheme, the collinear function will appear in the formulas of both the regions. In fact, it is common to write the cross section formula in the low- $q_{T}$ region as the Fourier Transform of the corresponding quantity in the conjugate $b_{T}$-space and expand the TMDs in the small- $b_{T}$ regime in an Operator Product Expansion (OPE), involving the collinear functions convoluted with some computable Wilson coefficient $[1,2,3])$. As a consequence, the collinear functions are of the utmost importance in the description of the whole $q_{T}$-spectrum of data and the impact of the uncertainty associated to their extraction should always be carefully taken into account.

\section{Large- $q_{T}$ region}

In the large- $q_{T}$ region, the SIDIS cross section can be written as [4]:

$$
\frac{d \sigma}{d x_{B j} d y d z_{h} d P_{T}^{2}}=\frac{\alpha_{S}}{\pi} \sum_{i j} \int_{x_{B j}}^{x_{M A X}} \frac{d x}{x} \int_{z_{h}}^{z_{M A X}} \frac{d z}{z} f_{i}\left(\frac{x_{B j}}{x}, Q^{2}\right) \frac{d \widehat{\sigma}_{i j}}{d x d y d z d\left(P_{T} / z_{h}\right)^{2}} D_{j}\left(\frac{z_{h}}{z}, Q^{2}\right),
$$

where the virtual photon has a transverse momentum given by $q_{T}=P_{T} / z_{h}$. Furthermore, $x_{B j}$ denotes the collinear momentum fraction carried by the initial parton with respect to the parent hadron and $z_{h}$ that of the outgoing hadron with respect to the fragmenting parton, while $d \widehat{\sigma}$ is the partonic cross-section of the process, which can be computed perturbatively in terms of the leptonic $L_{\mu \nu}$ and the hadronic $M_{\mu \nu}$ tensors:

$$
\frac{d \widehat{\sigma}_{i j}}{d x d y d z d\left(P_{T} / z_{h}\right)^{2}}=\frac{\alpha^{2}}{16 \pi^{2} Q^{4}} \frac{y}{z^{2}} \sum_{f} e_{f}^{2} L_{\mu v} M_{i j}^{\mu v}(x, y) \delta\left[z Q^{2}\left(\frac{\left(P_{T} / z_{h}\right)^{2}}{Q^{2}}-\frac{1-x}{x} \frac{1-z}{z}\right)\right] .
$$


The Dirac delta shapes the phase space integrals in such a way that the variables $x$ and $z$ never reach values greater than their allowed maxima. Since the most recent COMPASS data (Ref. [5]) are differential in $P_{T}^{2}$, the SIDIS cross section of Eq. 2.1 can be directly compared to data.

\subsection{Impact of the FFs set.}

Following Ref. [4], the formula for the cross section (Eq. 2.1) has been written factoring out one power of $\alpha_{S}$. In fact, in the high- $q_{T}$ regime, one should consider at least one real (gluon) emission, so that the cross section starts from first order in $\alpha_{S}$. Therefore, the natural choice for the collinear functions is a NLO set. However, as showed in Fig. 1, the data cannot be successfully described by using NLO PDFs and FFs sets, which significatively underestimate data. While reproducing the right shape seems not to be difficult, getting their right normalization is a problem. One might think that including the higher orders in the perturbative expansion of the partonic cross section could help in reaching a better agreement with experimental measurements. However, as shown in Ref. [8], even including $O\left(\alpha_{S}^{2}\right)$ corrections is not enough to reproduce the data. Having excluded the possibility of large contributions from higher order QCD corrections, it becomes crucial to evaluate the impact of the non-perturbative terms in the computation of the SIDIS cross section. As the PDFs are believed to be known to a rather good precision, we will focus on the FFs and assess how the uncertainties in their phenomenological extraction can affect the cross section itself. We will proceed by changing the FF set and try to shed some light on the impact of going

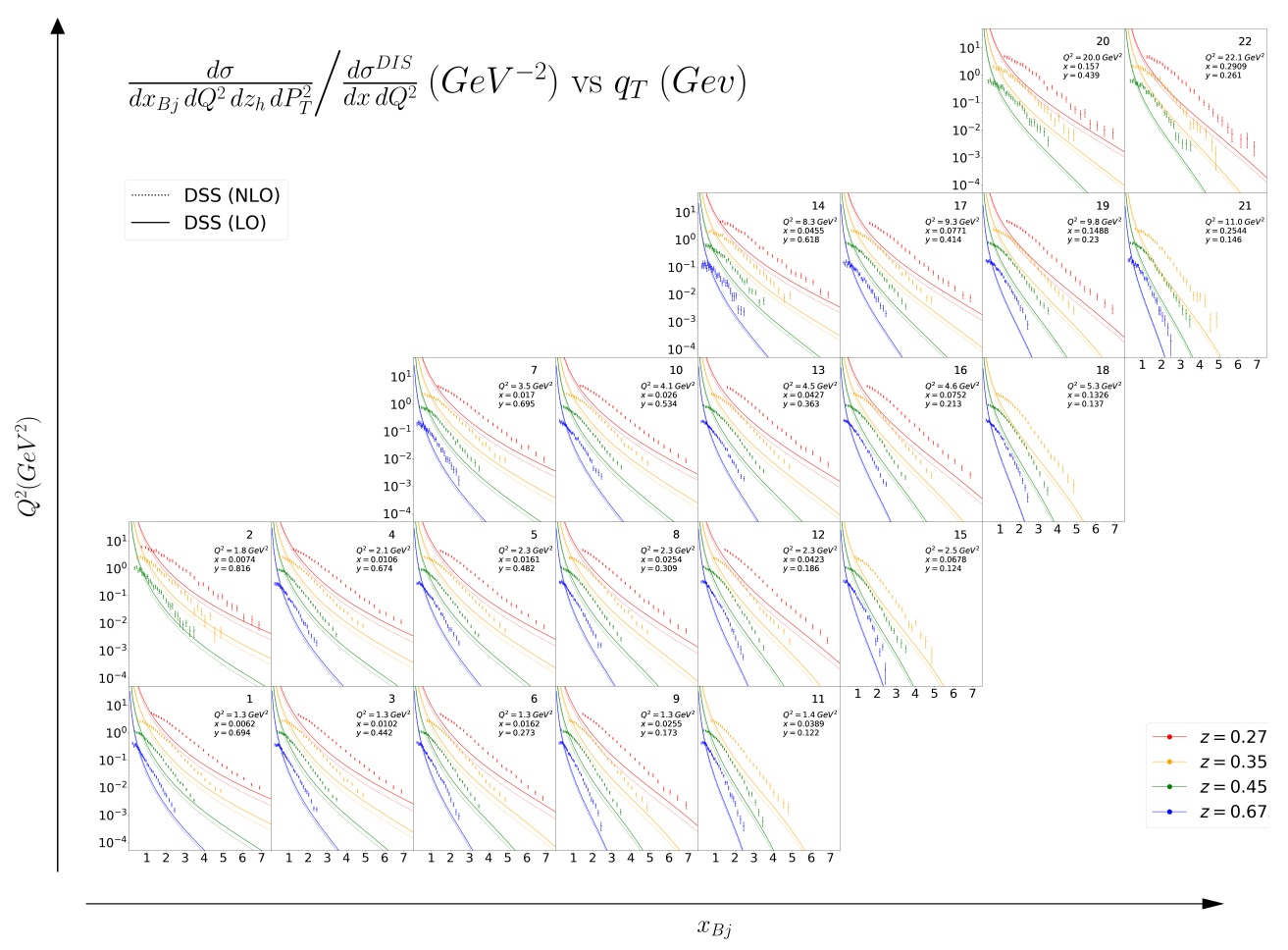

Figure 1: Differential multiplicities at COMPASS kinematic. Here we use the CT10NLO PDFs [6] and the FFs from Ref. [7]. The dotted lines correspond NLO, while the continuous lines to LO. 


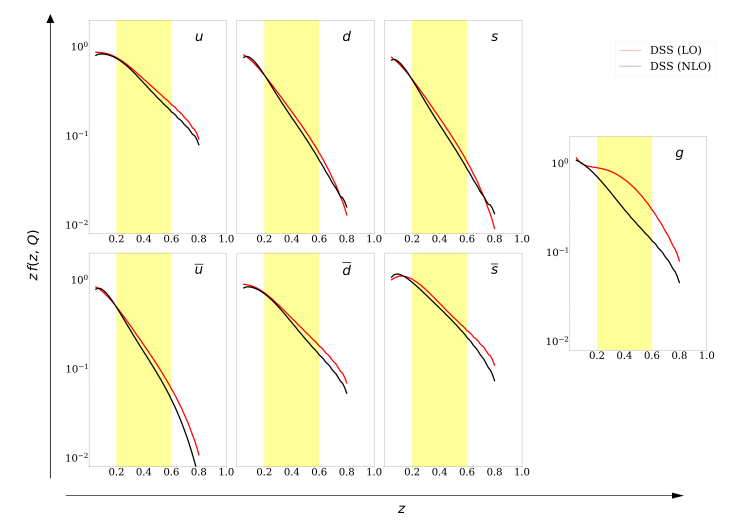

Figure 2: Comparison between DSS at LO (red lines) and DSS at NLO (black lines) at $Q^{2}=10 \mathrm{GeV}^{2}$.The yellow rectangles identify the range $0.2<z<0.6$, tipical of COMPASS kinematics. Notice that the gluon contribution at LO is considerably larger than at NLO in the selected kinematics, while no significative difference affects the quark and anti-quark contributions.

from LO to NLO, using a different set ${ }^{1}$ that provides larger values of the FFs for the COMPASS kinematics. Among the FFs, the gluon fragmentation function is the most difficult to determine, especially at low $Q$. Therefore, it is interesting to modify the FFs using a different set in which the gluon is overblown in the kinematics of interest. As shown in Fig. 2, the LO DSS set does the job: the multiplicities computed with this new set have the same shape of those shown in Fig. 1, but the discrepancy between theory and data is visibly reduced in size.

\subsection{Impact of uncertainties.}

The collinear functions are always provided with an uncertainty related to their extraction from experimental data ${ }^{2}$. Usually, the multiplicities are computed using only the central line of each set, however it is possible to include this uncertainty band on each theoretical curve, in order to investigate how much it affects the result. In particular, if these bands are large enough to cover the discrepancy between theory and data, it would be possible to choose collinear functions different from those of the central line, but still in agreement with experiments and consistent among each others.

The computation of the uncertainty bands has been implemented using the LHAPDF Python routine (Ref. [9]). As the uncertainties for DSS FF sets are not available, we used Neural Networks collinear functions, (Ref. [10,11]). Unfortunately, the NN FFs are provided only for pion production, while COMPASS multiplicities are given for unseparated charged hadrons. Hence the analysis does not involve the explicit comparison with experimental data, although it provides a reliable estimate of the sizes of the errors related to the collinear functions.

\footnotetext{
${ }^{1}$ Changing the functional form of a particular collinear function, e.g. the gluon alone, could be inconsistent with the other flavors, because of counting rules that relate them. Hence, to modify even just one collinear function, the whole set should be changed.

${ }^{2}$ In particular, this is the statistical error associated with the determination of the correct functional form of collinear PDFs and FFs
} 


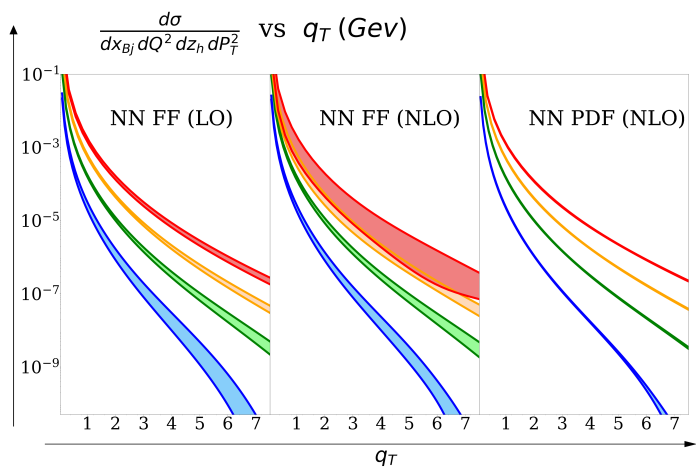

Figure 3: Uncertainty bands associated to the SIDIS cross section at high $q_{T}$ for $Q^{2}=2.1 \mathrm{GeV}^{2}, x=0.0106$ and $y=0.674$. The colors identify different values of $z$, as in Fig. 1. The most sizable contributions are due to the FFs, while the PDF bands are rather small. Note that although the NLO FFs uncertainties are larger than the their LO counterparts, their sizes do not seem to be able to cover the gap between theory and data.

\section{Low $-q_{T}$ region}

In the low- $q_{T}$ region it is still possible to write a factorized formula for SIDIS using a TMD factorization scheme, which now involves TMD distributions instead of collinear functions. In this case, the cross section is expressed as a Fourier transform from the conjugate $b_{T}$-space, where $b_{T}$ is the transverse separation of the operators defining the TMDs in coordinate space. Following Refs. [1, 4], we can write:

$$
\frac{d \sigma}{d x_{B j} d y d z_{h} d q_{T}^{2}}=\pi z^{2} H^{2}(Q, \mu) \int \frac{d^{2} \vec{b}_{T}}{(2 \pi)^{2}} e^{i \vec{q}_{T}} \cdot \vec{b}_{T}\left\{\sum_{j} e_{j}^{2} \widetilde{F}_{j}\left(x, b_{T}, \mu, \zeta_{F}\right) \widetilde{D}_{j}\left(z, b_{T}, \mu, \zeta_{D}\right)\right\} .
$$

The TMDs $\widetilde{F}$ and $\widetilde{D}$ are given by:

$$
\widetilde{F}_{j}\left(x, b_{T}, \mu, \zeta_{F}\right)=\widetilde{C}_{f}^{j}\left(\xi, b_{\star}, \mu_{b}, \zeta_{b}\right) \otimes f_{j}(\mu) \times e^{S_{\mathrm{pert}}\left(b_{\star}, Q / \mu_{b}, \zeta_{F} / \zeta_{b}\right)} \times e^{S_{\mathrm{NP}}\left(b_{T}\right)},
$$

where $b_{\star}\left(b_{T}\right)$ reproduces $b_{T}$ for small values of $b_{T}$ but is no larger that a certain $b_{\max }$ at large$b_{T}$, while the reference scales $\mu_{b} \propto 1 / b_{T}$ and $\zeta_{b}=\mu_{b}^{2}$ are the standard choices for the small- $b_{T}$ region. The perturbative Sudakov factor, $S_{\text {pert }}$, orginates from the contribution of soft gluons and from the anomalous dimensions, while a model, $S_{\mathrm{NP}}$, is needed to parametrize the intrinsecally non-perturbative large- $b_{T}$ region. The contribution of the collinear functions in Eq. 3.2 appears non-trivially and hence it is possible to study the impact of the error of the collinear functions in the TMD region. Similarly to what happens at large- $q_{T}$, also in the low- $q_{T}$ region there is a normalization problem that affects the agreement between theory and data. However, Fig. 4 shows that using a different set of FFs this problem becomes less severe. In fact, for NLO FFs the mismatch due to normalization is roughly a factor 2 , while using LO FFs one can gain a factor $\sim 5$ in the low- $q_{T}$ region. However, the uncertainty band associated to the NLO FFs is so large that not only the central line of the LO FFs set, but also its full error band, is totally included.

In conclusion, this study shows that the impact of the uncertainties on the FF extraction may well be large enough to allow for a better description of the experimental data, even preserving consistency among flavors. 


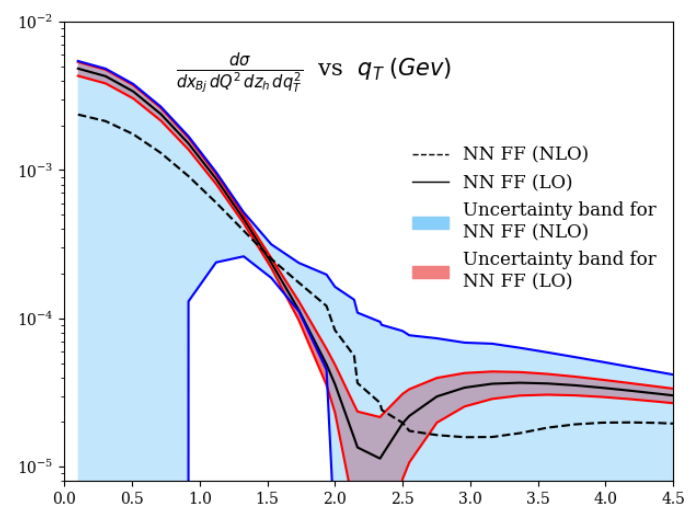

Figure 4: Uncertainties due to the collinear FFs on the cross section at low- $q_{T}\left(Q^{2}=2.1 \mathrm{GeV}^{2}, x=0.0106\right.$, $y=0.674, z=0.35$ ). The PDF set is from Ref. [10]. The solid (black) line represents the cross section, Eq. 3.1, as obtained by using the LO FF set from Ref. [11] and the red band is its corresponding uncertainty, while the dashed line represents the result obtained by using the NLO FF set, with its uncertainty band (in blue).

\section{References}

[1] J. Collins, Foundations of perturbative QCD, Camb. Monogr. Part. Phys. Nucl. Phys. Cosmol. 32 (2011) 1.

[2] J. Collins and T. Rogers, Understanding the large-distance behavior of transverse-momentum-dependent parton densities and the Collins-Soper evolution kernel, Phys. Rev. D91 (2015) 074020 [1412.3820].

[3] J. Collins, L. Gamberg, A. Prokudin, T. C. Rogers, N. Sato and B. Wang, Relating Transverse Momentum Dependent and Collinear Factorization Theorems in a Generalized Formalism, Phys. Rev. D94 (2016) 034014 [1605.00671].

[4] M. Anselmino, M. Boglione, A. Prokudin and C. Turk, Semi-Inclusive Deep Inelastic Scattering processes from small to large P(T), Eur. Phys. J. A31 (2007) 373 [hep-ph/ 0606286 ].

[5] COMPASS collaboration, Transverse-momentum-dependent Multiplicities of Charged Hadrons in Muon-Deuteron Deep Inelastic Scattering, Phys. Rev. D97 (2018) 032006 [1709.07374].

[6] H.-L. Lai, M. Guzzi, J. Huston, Z. Li, P. M. Nadolsky, J. Pumplin et al., New parton distributions for collider physics, Phys. Rev. D 82 (2010) 074024.

[7] D. de Florian, R. Sassot and M. Stratmann, Global analysis of fragmentation functions for pions and kaons and their uncertainties, Phys. Rev. D 75 (2007) 114010.

[8] J. O. Gonzalez-Hernandez, T. C. Rogers, N. Sato and B. Wang, Challenges with large transverse momentum in semi-inclusive deeply inelastic scattering, Phys. Rev. D 98 (2018) 114005.

[9] A. Buckley, J. Ferrando, S. Lloyd, K. Nordström, B. Page, M. Rüfenacht et al., LHAPDF6: parton density access in the LHC precision era, Eur. Phys. J. C75 (2015) 132 [1412 . 7420 ].

[10] R. D. Ball, V. Bertone, S. Carrazza, C. S. Deans, L. D. Debbio, S. Forte et al., Parton distributions with lhc data, Nuclear Physics B 867 (2013) 244

[11] V. Bertone, S. Carrazza, N. P. Hartland, E. R. Nocera and J. Rojo, A determination of the fragmentation functions of pions, kaons, and protons with faithful uncertainties, The European Physical Journal C 77 (2017) 516. 